\author{
Hubert J. M. Geijselaers \\ University of Twente, \\ P.0. Box 217 \\ 7500 AE Enschede, \\ The Netherlands \\ e-mail: h.j.m.geijselaers@wb.utwente.nl \\ Annette J. E. Koning \\ Mecal BV, \\ P.0. Box 286 \\ 7500 AG Enschede, \\ The Netherlands \\ e-mail: A.Koning@mecal.nl
}

\section{Finite Element Analysis of Thermoelastic Instability With Intermittent Contact}

\begin{abstract}
The equations that describe the development of corrugations on block braked wheel treads caused by thermoelastic instability are discretized using the finite element method. The perturbations of temperatures and distortions are described by an amplitude function, which is spatially fixed multiplied by a sinusoidal running wave term of fixed wavelength. The governing equations are such that the wave term cancels out. Only the amplitude functions are discretized in the finite element model. The intermittent nature of the contact is directly specified through the boundary conditions. Results are obtained for a simplified two-dimensional model of a train wheel. These results agree with analytical results.
\end{abstract}

\section{Introduction}

The study reported on here is motivated by the occurrence of corrugations on freight train wheels. Freight trains are fitted with block brakes. During braking it is observed that hot spots develop on the wheel treads (Hiensch, 1998). The hot spots are stationary with respect to the wheel. After braking, the wheels are left with a corrugation with a wavelength of approximately $60 \mathrm{~mm}(\approx 48$ waves around the circumference) and an amplitude of a few microns. This causes a considerable increase in noise production during normal train operation. Dittrich et al. (1994) examined the wheel roughness and sound emission of wheel sets fitted with four different types of braking systems. They found that the roughness of block braked trains with cast iron brakes is considerably higher than the roughness of disc braked trains.

In this paper it is demonstrated how the finite element method may be used to simulate the development of hot spots on block braked wheel treads. It is assumed that the phenomenon is caused by thermoelastic instability. Thermoelastic instability has been extensively studied over the last 30 years among others, by Burton et al. (1973), Kennedy (1980) and with special reference to brake systems by Anderson and Knapp (1990) and Barber et al. (1985). From the last group a number of papers deal with the problem of thermoelastic instability under conditions of intermittent contact. A Fourier series approach is used (Ruiz-Ayala et al., 1996) and an approximation, based on the ratio of contact length to total circumference is derived.

In this paper we apply the finite element method to analyze intermittent contact. However, unlike Du et al. (1997) we do not calculate temperature and distortion perturbations proper but amplitude functions of the distortion and temperature perturbations as postulated in Burton's method.

The derivation presented here is restricted to the equations of the wheel. Extension to distortion of the brake blocks seems straight forward (Koning and Geijselaers, 1999) but has not been implemented yet. Rather, the brake blocks are modeled as rigid and nonconducting, which means, that their presence can be (conveniently) accounted for through the boundary conditions. Flexibility of the brake blocks can still be approximately taken into account by using a lower equivalent stiffness for the wheel material.

\section{Equations}

The formulation of the governing equations is done in a reference frame, that moves with the wheel, but does not rotate, see Fig. 1. The advantage is that the boundary conditions due to the brake

Contributed by the Tribology Division of The American Society of Mechanical ENGINEERS and presented at the STLE/ASME Tribology Conference, Orlando, FL, October 10-13, 1999. Manuscript received by the Tribology Division May 4, 1999; revised manuscript received July 21, 1999. Paper No. 99-Trib-43. Associate Technical Editor: M. B. Bryant. blocks remain at a fixed position, while the resulting equations still remain relatively simple. The equations are derived in a rectangular coordinate system where the $x$-coordinate runs along the tread surface and the $y$-coordinate points outward. This is motivated by the very small penetration depth of the thermal perturbation field. The wheel has a velocity $V$ with respect to the $x$-coordinate.

2.1 Thermal Equations. The thermal equation for the wheel contains a convective term:

$$
\rho c_{v}\left(\frac{\partial T}{\partial t}+V \frac{\partial T}{\partial x}\right)-k\left(\frac{\partial^{2} T}{\partial x^{2}}+\frac{\partial^{2} T}{\partial y^{2}}\right)=0
$$

The boundary conditions, where the wheel and blocks contact, are:

$$
k \frac{\partial T}{\partial y}=q
$$

Here $\rho c_{v}$ and $k$ are the heat capacity and thermal conductivity of the wheel material, $q$ is the heat dissipated in the contact.

In order to study thermoelastic instability a corrugation of the wheel is assumed, giving rise to a perturbation of the heat dissipation $q$ :

$$
\begin{aligned}
& q(x, t)= q_{1}(x) \cos \left(\frac{n}{R}(x-V t)\right)+q_{2}(x) \sin \left(\frac{n}{R}(x-V t)\right) \\
&=\mu V p_{1}(x) \cos \left(\frac{n}{R}(x-V t)\right) \\
& \quad+\mu V p_{2}(x) \sin \left(\frac{n}{R}(x-V t)\right)
\end{aligned}
$$

Here $\mu$ is the friction coefficient and $p$ is the contact pressure between brake block and wheel tread. Likewise a solution for the perturbation of the temperatures is assumed as:

$$
\begin{aligned}
T(x, y, t)=T_{1}(x, y) \cos ( & \left.\frac{n}{R}(x-V t)\right) \\
& +T_{2}(x, y) \sin \left(\frac{n}{R}(x-V t)\right)
\end{aligned}
$$

Substitution of this into Eq. (1) yields:

$$
\begin{array}{r}
\cos \left(\frac{n}{R}(x-V t)\right)\left\{\rho c_{v} V \frac{\partial T_{1}}{\partial x}-k\left(\frac{\partial^{2} T_{1}}{\partial x^{2}}+\frac{2 n}{R} \frac{\partial T_{2}}{\partial x}-\frac{n^{2}}{R^{2}} T_{1}\right.\right. \\
\left.\left.+\frac{\partial^{2} T_{1}}{\partial y^{2}}\right)\right\}+\sin \left(\frac{n}{R}(x-V t)\right)\left\{\rho c_{v} V \frac{\partial T_{2}}{\partial x}-k\left(\frac{\partial^{2} T_{2}}{\partial x^{2}}\right.\right. \\
\left.\left.-\frac{2 n}{R} \frac{\partial T_{1}}{\partial x}-\frac{n^{2}}{R^{2}} T_{2}+\frac{\partial^{2} T_{2}}{\partial y^{2}}\right)\right\}=0
\end{array}
$$



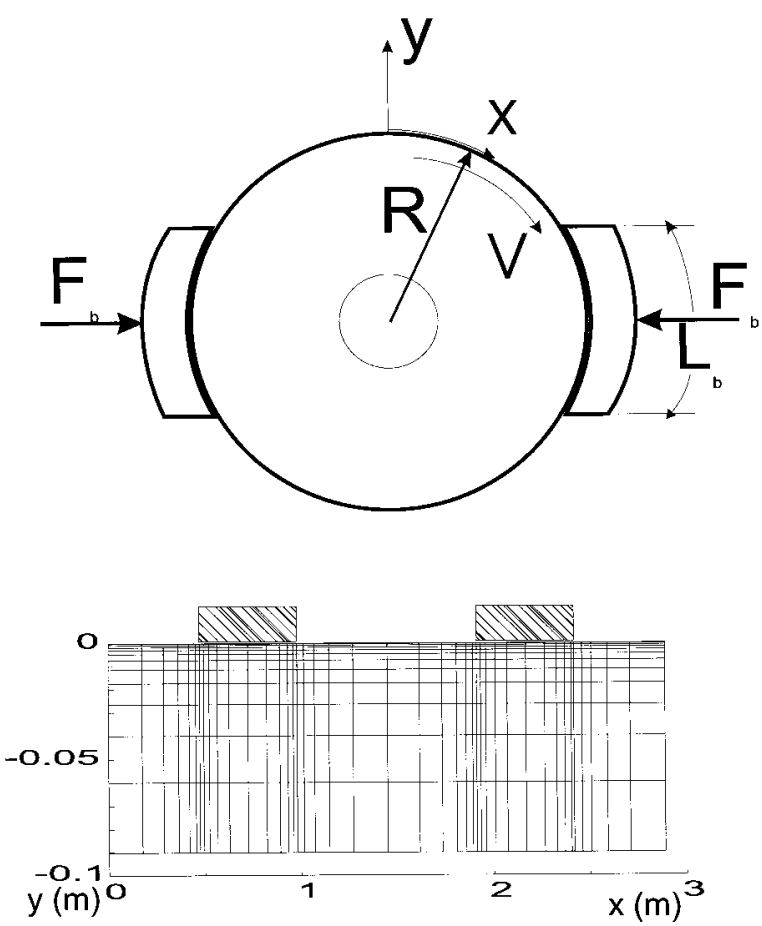

Fig. 1 The geometry and mesh of the block braked train wheel

For this to be valid, the terms between braces have to be zero. This results in two coupled equations:

$$
\begin{aligned}
& \rho c_{v} V \frac{\partial T_{1}}{\partial x}-k\left(\frac{\partial^{2} T_{1}}{\partial x^{2}}+\frac{2 n}{R} \frac{\partial T_{2}}{\partial x}-\frac{n^{2}}{R^{2}} T_{1}+\frac{\partial^{2} T_{1}}{\partial y^{2}}\right)=0 \\
& \rho c_{v} V \frac{\partial T_{2}}{\partial x}-k\left(\frac{\partial^{2} T_{2}}{\partial x^{2}}-\frac{2 n}{R} \frac{\partial T_{1}}{\partial x}-\frac{n^{2}}{R^{2}} T_{2}+\frac{\partial^{2} T_{2}}{\partial y^{2}}\right)=0
\end{aligned}
$$

The boundary conditions to these equations follow from Eq. (2):

$$
\begin{gathered}
\text { in the contact }\left\{\begin{array}{c}
k \frac{\partial T_{1}}{\partial y}=q_{1} \\
k \frac{\partial T_{2}}{\partial y}=q_{2}
\end{array}\right. \\
\text { outside the contact }\left\{\begin{array}{c}
k \frac{\partial T_{1}}{\partial y}=0 \\
k \frac{\partial T_{2}}{\partial y}=0
\end{array}\right.
\end{gathered}
$$

2.2 Simplification. The perturbation is described with an amplitude and a harmonic term. The derivatives of the harmonic term give rise to expressions containing the parameter $n / R$. A corrugation on the wheel tread typically has a wave length between 65 and $130 \mathrm{~mm}$. This means that the wave length related parameter $n / R$ has a typical value of $0.1 \mathrm{~mm}^{-1}$. The amplitude functions $T_{i}$, $u_{i}$ etc. are related to the position of the brake blocks. This means that for the derivatives of these functions holds that $\partial T_{i} / \partial x=$ $O(2 / R) T_{i}$. From this it is apparent that the terms containing $n / R$ are at least an order bigger than the derivatives with respect to $x$. The obvious step is to neglect these derivatives in comparison to the terms with $n / R$. As a result all equations which have a coupling between the ()$_{1}$ field and the ()$_{2}$ field become uncoupled. There exists only a coupling through the shear traction under the brake block. The effect of this coupling was investigated by Lee and Barber (1993) and was found negligible. For this reason we neglect this coupling and restrict ourselves to the ()$_{1}$ field.

$$
\begin{aligned}
& q(x, t)=q_{1}(x) \cos \left(\frac{n}{R}(x-V t)\right) \\
& T(x, t)=T_{1}(x) \cos \left(\frac{n}{R}(x-V t)\right)
\end{aligned}
$$

2.3 Elasticity Equations. The perturbation to the thermal field is assumed to cause a conforming perturbation to the distortions. The displacements of the wheel material with respect to the unperturbed configuration are described by the $x$ - and $y$-components $u(x, y, t)$ and $v(x, y, t)$ belonging to the $\cos (n /$ $R(x-V t))$ terms of the thermal field:

$$
\begin{aligned}
& u(x, y, t)=u_{1}(x, y) \sin \left(\frac{n}{R}(x-V t)\right) \\
& v(x, y, t)=v_{1}(x, y) \cos \left(\frac{n}{R}(x-V t)\right)
\end{aligned}
$$

The deformations are linked to the distortions by the straindisplacement relations, which are simplified like in Section 2.2:

$$
\begin{gathered}
\epsilon_{x x}(x, y, t)=\frac{\partial u}{\partial x} \approx \frac{n}{R} u_{1} \cos \left(\frac{n}{R}(x-V t)\right) \\
\epsilon_{y y}(x, y, t)=\frac{\partial v}{\partial y}=\frac{\partial v_{1}}{\partial y} \cos \left(\frac{n}{R}(x-V t)\right) \\
\gamma_{x y}(x, y, t)=\frac{\partial u}{\partial y}+\frac{\partial v}{\partial x} \approx\left(\frac{\partial u_{1}}{\partial y}-\frac{n}{R} v_{1}\right) \sin \left(\frac{n}{R}(x-V t)\right)
\end{gathered}
$$

\section{Nomenclature}

$A_{i j}=$ heat capacity matrix

$c_{v}=$ specific heat capacity

$C_{i j}=$ conduction matrix

$E=$ elasticity modulus

$F_{1 i}=$ force vector

$k=$ thermal conduction coefficient

$K_{i j}=$ stiffness matrix

$L_{b}=$ Length of one brake block

$n=$ number of waves about the circumference

$N=$ interpolation function

$p=$ contact pressure

$p_{1}=$ amplitude function of contact pressure perturbation

$P_{i j}=$ thermo-mechanical influence matrix

$q=$ surface heat input $q_{1}=$ amplitude function of heat input perturbation

$Q_{1 i}=$ heat input vector

$R=$ wheel radius

$R_{i j}=$ thermo-mechanical coupling matrix

$t=$ time

$T=$ temperature

$T_{1}=$ amplitude function of temperature perturbation

$u=x$-displacement

$u_{1}=$ amplitude function of the $x$-displacement perturbation

$v=y$-displacement

$v_{1}=$ amplitude function of the $y$-displacement perturbation

$V=$ sliding velocity
$V_{0}=$ critical velocity at full length contact

$V_{c r}=$ critical velocity

$x=$ coordinate along sliding plane

$y=$ coordinate perpendicular to sliding plane

$\alpha=$ thermal expansion coefficient

$\gamma_{x y}=$ shear strain

$\boldsymbol{\epsilon}_{\alpha \beta}=$ strain tensor component

$\mu=$ friction coefficient

$\nu=$ Poisson ratio

$\rho=$ material density

$\sigma_{\alpha \beta}=$ stress tensor component

$\sigma_{\alpha \beta 1}=$ amplitude function of the stress perturbation 
The stresses that occur due to the distortions are assumed to have a similar form as the temperature and distortion fields.

$$
\begin{aligned}
& \sigma_{x x}(x, y, t)=\sigma_{x x 1}(x, y) \cos \left(\frac{n}{R}(x-V t)\right) \\
& \sigma_{y y}(x, y, t)=\sigma_{y y 1}(x, y) \cos \left(\frac{n}{R}(x-V t)\right) \\
& \sigma_{x y}(x, y, t)=\sigma_{x y 1}(x, y) \sin \left(\frac{n}{R}(x-V t)\right)
\end{aligned}
$$

Substitution into the equilibrium equations, while again applying the simplifications according to Section 2.2, yields two coupled equations.

$$
\begin{gathered}
-\frac{n}{R} \sigma_{x x 1}+\frac{\partial \sigma_{x y 1}}{\partial y}=0 \\
\frac{n}{R} \sigma_{x y 1}+\frac{\partial \sigma_{y y 1}}{\partial y}=0
\end{gathered}
$$

The boundary conditions at the wheel surface $y=0$ are:

$$
\begin{aligned}
& \text { in the contact }\left\{\begin{array}{l}
\sigma_{y y 1}=-p_{1} \\
v_{1}=0 \\
\sigma_{x y 1}=0
\end{array}\right. \\
& \text { outside the contact }\left\{\begin{array}{l}
\sigma_{y y 1}=0 \\
\sigma_{x y 1}=0
\end{array}\right.
\end{aligned}
$$

The amplitude functions of the stress perturbations depend on the strain and the temperature perturbations.

$$
\begin{array}{r}
\left\{\begin{array}{c}
\sigma_{x x} \\
\sigma_{y y} \\
\sigma_{x y}
\end{array}\right\}=\frac{E}{(1+\nu)(1-2 \nu)}\left[\begin{array}{ccc}
1-\nu & \nu & 0 \\
\nu & 1-\nu & 0 \\
0 & 0 & \frac{1-2 \nu}{2}
\end{array}\right] \\
\times\left\{\begin{array}{c}
\epsilon_{x x} \\
\epsilon_{y y} \\
\gamma_{x y}
\end{array}\right\}-\frac{\alpha E}{1-2 \nu}\left\{\begin{array}{c}
1 \\
1 \\
0
\end{array}\right\} T
\end{array}
$$

This is the constitutive equation for plane strain, which applies to perturbations with wavelengths of the order of the wheel tread width.

\section{Numerical Discretization}

The thermal balance equation (6) and the boundary conditions Eq. (7) are written in the weak form using the SUPG technique (Brooks and Hughes, 1982). Bilinear quadrilateral elements are used. The following matrix equation is found:

$$
V[A]\left\{T_{1}\right\}+[C]\left\{T_{1}\right\}=\left\{Q_{1}\right\}
$$

The components of the matrices $[A],[C]$ and vector $\left\{Q_{1}\right\}$ are given in appendix A. A similar procedure is applied to the mechanical equilibrium equation (12) and the boundary conditions Eq. (13) which results in a matrix equation:

$$
[K]\left\{u_{1}\right\}+[R]\left\{T_{1}\right\}=\left\{F_{1}\right\}
$$

At the onset of instability, the presence of the brake blocks suppresses the displacement perturbation $v_{1}(x, 0)$ under the brake blocks. When this is taken into account, a relation between the pressures $p_{1}(y)$ in the contact region and the temperature perturbations can be derived. To this end the set of displacement degrees of freedom is partitioned into the degrees of freedom suppressed by the brake blocks (index $s$ ) and the remaining degrees of freedom (index $f$ ).

$$
\left[\begin{array}{cc}
K_{s s} & K_{s f} \\
K_{f s} & K_{f f}
\end{array}\right]\left\{\begin{array}{l}
u_{1 s} \\
u_{1 f}
\end{array}\right\}+\left[\begin{array}{l}
R_{s} \\
R_{f}
\end{array}\right]\left\{T_{1}\right\}=\left\{\begin{array}{c}
F_{1 s} \\
0
\end{array}\right\}
$$

Substitution of $u_{1 s}=0$ and elimination of $u_{1 f}$ yields for $\left\{F_{1 s}\right\}$ :

$$
\left\{F_{1 s}\right\}=\left[\left[R_{s}\right]-\left[K_{s f}\right]\left[K_{f f}^{-1}\right]\left[R_{f}\right]\right]\left\{T_{1}\right\}=\left[P_{s}\right]\left\{T_{1}\right\}
$$

The vector $\left\{F_{1 s}\right\}$ contains the surface pressures $p_{1}(x)$ under the brake block. The heat input $\left\{Q_{1}\right\}$ depends on the surface pressures:

$$
q_{1}(x)=\mu p_{1} V
$$

This means, that $\left\{Q_{1}\right\}$ can be written as:

$$
\left\{Q_{1}\right\}=-\mu V[L]\left\{F_{1 s}\right\}=-\mu V[L]\left[P_{s}\right]\left\{T_{1}\right\}
$$

Here $[L]$ is a selection matrix. Substitution into Eq. (15) yields an eigenvalue problem:

$$
\left[V\left[[A]+\mu[L]\left[P_{s}\right]\right]+[C]\right]\left\{T_{1}\right\}=0
$$

The critical velocity $V_{c r}$ is found as the lowest real positive eigenvalue.

\section{Results}

The analytical solution for the critical velocity of an elastic body rubbing against a rigid, nonconducting half space is:

$$
V_{c r}=2(1-\nu) \frac{k n}{R \mu E \alpha}
$$

For the situation with 48 waves around the circumference, using material properties as in Appendix B, the critical velocity is: $V_{c r}=$ $0.0248 \mathrm{~ms}^{-1}$.

The numerical model gives a converged solution identical up to four relevant decimals to this analytical result when a depth of one and a half times the wavelength is modeled with 12 elements.

4.1 Calculations on Tread Braked Wheels. An element mesh is used with 42 elements in the $x$-direction and 12 elements in the $y$-direction (Fig. 1). In the $x$-direction, the elements are refined toward the ends of the block brakes. In the $y$-direction, element refinement is applied toward the tread surface. The penetration depth depends on the number of waves on the circumference. A depth of one and a half times the wavelength is modeled.

The number of eigenvalues is equal to the number of nodes in the model. Most of these eigenvalues are complex, having a real as well as an imaginary part. In Fig. 2 the position of some of the eigenvalues is displayed; a number of negative eigenvalues are not displayed because they are out of the range. Only one eigenvalue is real and positive, the remainder is negative real or complex. Since negative velocities are not realistic, these are left out of consideration. The complex eigenvalues have no physical meaning.

In the calculation with 48 waves around the circumference the positive real eigenvalue has a value of 0.0577 . By means of the eigenvectors, the amplitudes of the perturbations of the temperature field, the displacement fields and the contact pressure at the tread surface can be determined. In Fig. 3 through 6 these amplitude functions are displayed. In Fig. 6 the amplitude of the pressure perturbation on the tread surface, where the $y$-displacement is suppressed underneath the brake blocks, is shown.

A number of calculations is done where the length of the brake block is varied. The results are shown in Figure 7 together with the tendency as expected from the analytical results from Ruiz-Ayala et al. (1996) and Barber et al. (1985). The numerical results match very well. Due to the nature of the finite element method an overestimation of the stiffness of the system is expected, resulting in an underestimation of the critical velocity. This shows up as a higher value of $V_{0} / V_{c r}$ for the higher dimensionless contact lengths. With a low value of the dimensionless contact length the 


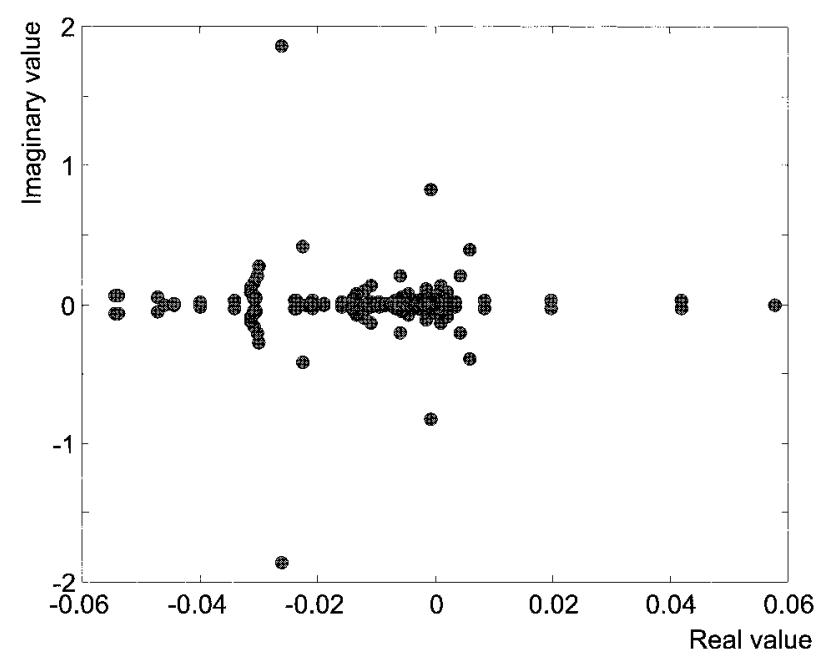

Fig. 2 The locations of the eigenvalues (48 waves)

critical speed increases which makes Barber et al. (1985) more applicable.

\section{Conclusions}

A method is presented to calculate thermoelastic instability during braking using a finite element discretisation.

The perturbations to the temperatures and distortions are written as the product of amplitude functions and a harmonic term. The amplitude functions are stationary with respect to the brakes. The harmonic term represents a running wave. The governing equa-

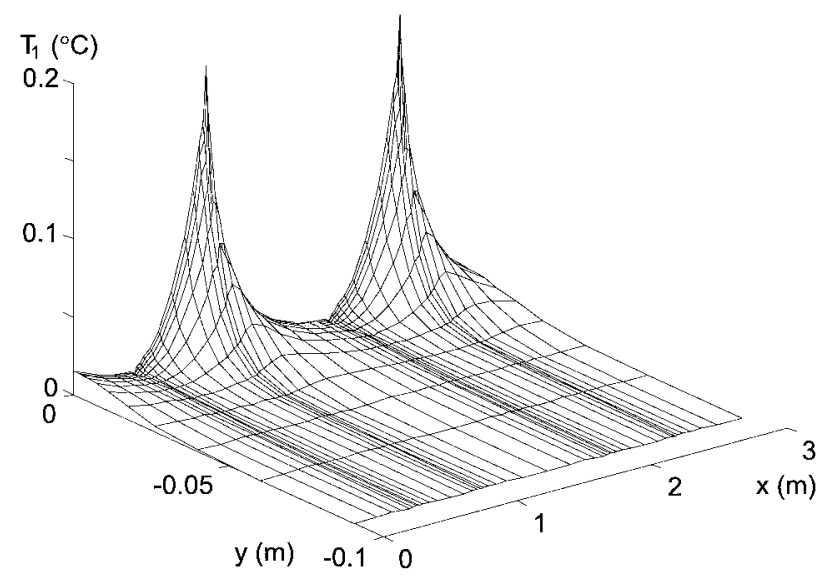

Fig. 3 Amplitude field of the temperature perturbation (48 waves)

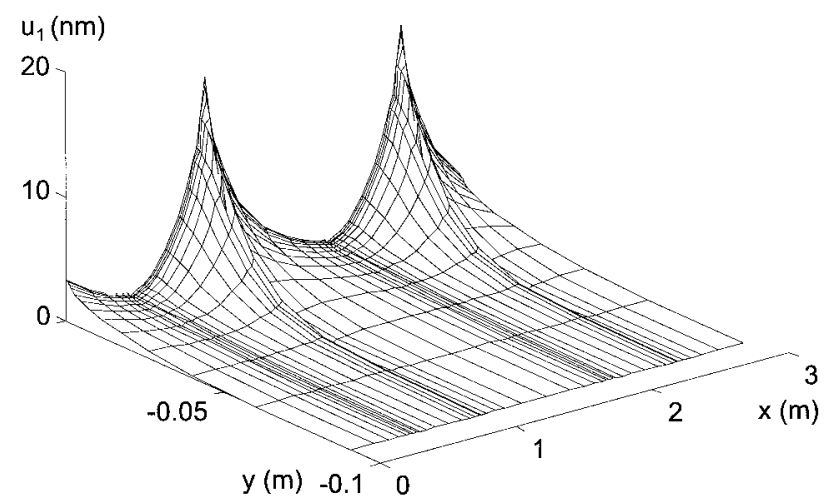

Fig. 4 Amplitude field of the $x$-displacement perturbation (48 waves)

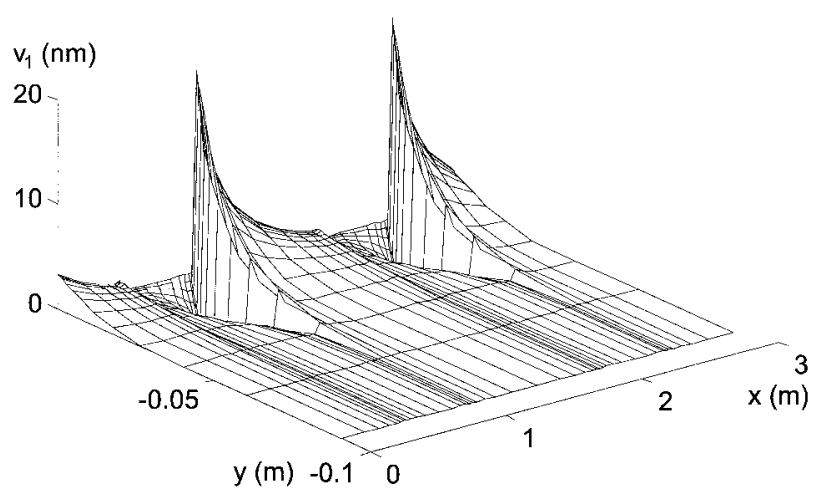

Fig. 5 Amplitude field of the $y$-displacement perturbation (48 waves)

tions are such, that the wave term cancels out. Only the amplitude function is discretized. The presence of the brakes is conveniently modeled through stationary boundary conditions.

Analytical results are reproduced well. The advantage is however, that calculations on configurations, which can not be tackled analytically, are also possible. Extension of this method to include the brake blocks is currently on its way.

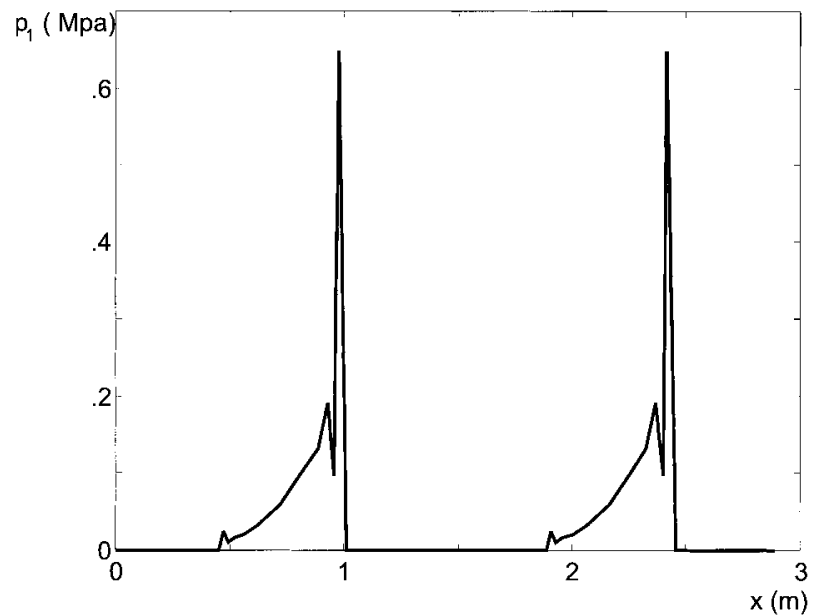

Fig. 6 Amplitude of the pressure perturbation on the tread surface (48 waves)

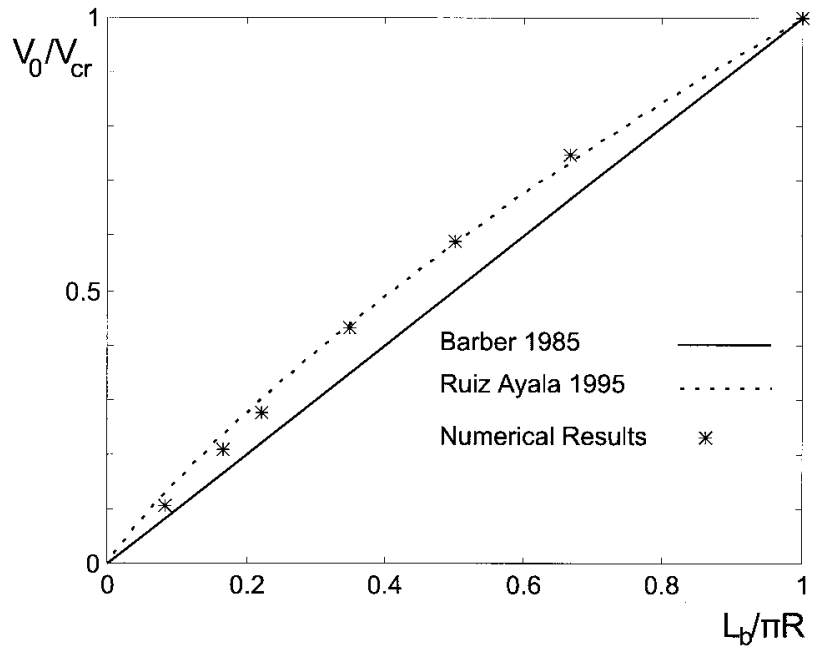

Fig. 7 Effect of the dimensionless contact length $L_{b} / \pi R$ on the critical speed (48 waves) 


\section{Acknowledgment}

This study is done within the context of the project ICES Stiller Treinverkeer (ICES-STV). Within the ICES-STV project the origin, the transfer and the measurement of railway noise is studied. The object is to increase the knowledge in this area in The Netherlands. This project is funded by the government of The Netherlands (EZ, VROM and V\&W) as well as by the institutes and companies that participate in it. The authors thank the funding parties for enabling this study.

\section{References}

Anderson, A., and Knapp, R., 1990, "Hotspotting in Automotive Friction Systems," Wear, Vol. 135, pp. 319-337.

Barber, J., Beamond, T., Waring, J., and Prichard, C., 1985, "Implications of Thermoelastic Instability for the Design of Brakes," ASME Journal of TriBology, Vol. 107, pp. 206-210.

Brooks, A., and Hughes, T., 1982, "Streamline Upwind Petrov Galerkin Formulations for Convection dominated Flows with particular emphasis on the Incompressible Navier-Stokes Equation," Comp. Meth Appl. Mech. Engrg., Vol. 32, pp. 199-259.

Burton, R., Nerlikar, V., and Kilaparti, S., 1973, "Thermoelastic Instability in a Seal-like Configuration," Wear, Vol. 24, pp. 177-188.

Dittrich, M., Biegstraten, F., Dings, P., and Thompson, D., 1994, "Wheel Roughness and Railway Noise," Rail Engineering International, Vol. 3, pp. 17-20.

Du, S., Zagrodzki, P., Barber, J., and Hulbert, G., 1997, "Finite Element Analysis of Frictionally Excited Thermoelastic Instability," Journal of Thermal Stresses, Vol. 20, pp. 185-201.

Hiensch, E., 1998, "Lasergecladde Wielen, Thermische Data Remproeven," Technical Report NSTO/98/9620329/012, NS Technisch Onderzoek.

Kennedy, F., 1980, "Thermo-Mechanical Phenomena in High Speed Rubbing," Wear, Vol. 59(2), pp. 149-163.

Koning, A., and Geijselaers, H. J. M., 1999, "Corrugations on the Block Braked Wheel Treads of Freight Trains," Technical Report WB.99/TMK-3589, Univ of Twente, The Netherlands.

Lee, K., and Barber, J., 1993, "The effect of Shear Tractions on Frictionally Excited Thermoelastic Instability,” Wear, Vol. 160, pp. 237-242.

Ruiz Ayala, J., Lee, K., Rahman, M., and Barber, J., 1996, "Effect of Intermittent Contact on the Stability of Thermoelastic Sliding Contact," ASME Journal of Tribology, Vol. 118, pp. 102-108.

\section{A P P E N D I X A}

\section{Systems Matrices}

The matrices $[A],[C]$ and the vector $\left\{Q_{1}\right\}$ are calculated as:

$$
\begin{gathered}
A_{i j}=\rho c_{v} \iint_{A}\left(N^{i}+\tau V \frac{\partial N^{i}}{\partial x}\right) \frac{\partial N^{j}}{\partial x} d x d y \\
C_{i j}=k \iint_{A}\left\{\left(N^{i}+\tau V \frac{\partial N^{i}}{\partial x}\right) \frac{n^{2}}{R^{2}} N^{j}+\frac{\partial N^{i}}{\partial y} \frac{\partial N^{j}}{\partial y}\right. \\
\left.-\tau V \frac{\partial N^{i}}{\partial x} \frac{\partial^{2} N^{j}}{\partial y^{2}}\right\} d x d y \\
Q_{1 i}=\int_{X_{b}} N^{i} q_{1}(x) d x
\end{gathered}
$$

$N^{k}(x, y)$ Are the shape functions. For the time parameter $\tau$ in (Brooks and Hughes, 1982) a value of $\tau=0.2 l_{e l} /|V|$ is recommended.

The matrices $[K],[R]$ and the vector $\left\{F_{1}\right\}$ are build up out of submatrices:

$$
\begin{gathered}
{\left[\begin{array}{ll}
K_{x x} & K_{x y} \\
K_{x y} & K_{y y}
\end{array}\right]_{i j}} \\
=\frac{E}{(1+\nu)(1-2 \nu)} \iint_{A}\left[B^{i}\right]^{T}\left[\begin{array}{ccc}
1-\nu & \nu & 0 \\
\nu & 1-\nu & 0 \\
0 & 0 & \frac{1-2 \nu}{2}
\end{array}\right] \\
\left\{\begin{array}{c}
R_{x} \\
R_{y}
\end{array}\right\}_{i k}=-\frac{\alpha E}{1-2 \nu} \iint^{j}\left[B^{i}\right]^{T}\left\{\begin{array}{c}
1 \\
1 \\
0
\end{array}\right\} N^{k} d x d y \\
\left\{\begin{array}{c}
F_{x} \\
F_{y}
\end{array}\right\}_{i}=\int_{X_{b}}\left[\begin{array}{cc}
N^{i} & 0 \\
0 & N^{i}
\end{array}\right]\left\{\begin{array}{c} 
\\
-p_{1}(x)
\end{array}\right\} d x
\end{gathered}
$$

The matrix $\left[B^{k}\right]$ describes the relation between strain amplitudes and nodal displacements:

$$
\begin{aligned}
\left\{\begin{array}{l}
\boldsymbol{\epsilon}_{x x 1} \\
\boldsymbol{\epsilon}_{y y 1} \\
\gamma_{x y 1}
\end{array}\right\} & =\sum_{k=1}^{N_{k}}\left[\begin{array}{cc}
\frac{n}{R} N^{k} & 0 \\
0 & \frac{\partial N^{k}}{\partial y} \\
\frac{\partial N^{k}}{\partial y} & -\frac{n}{R} N^{k}
\end{array}\right]\left\{\begin{array}{c}
u_{1}^{k} \\
v_{1}^{k}
\end{array}\right\} \\
& =\sum_{k=1}^{n}\left[B^{k}\right]\left\{\begin{array}{c}
u_{1}^{k} \\
v_{1}^{k}
\end{array}\right\}
\end{aligned}
$$

\section{A P P E N D I X B}

\section{Material Properties and Geometry}

Geometry and material properties used correspond to those of wheel type Volwiel (Ø920 mm) Type 24 material code R7:

Diameter

Brake Block Length

Density

Heat Conduction Coefficient

Specific Heat Capacity

Thermal Expansion Coefficient

Elasticity Modulus

Poison Ration

Friction Coefficient

$$
\begin{aligned}
& D=920(\mathrm{~mm}) \\
& L_{b}=505(\mathrm{~mm}) \\
& \rho=7830\left(\mathrm{~kg} / \mathrm{m}^{3}\right) \\
& k=47(\mathrm{~W} / \mathrm{mK}) \\
& c_{v}=445(\mathrm{~J} / \mathrm{kgK}) \\
& \alpha=11 * 10^{-6}\left(\mathrm{~K}^{-1}\right) \\
& E=210 * 10^{9}\left(\mathrm{~N} / \mathrm{m}^{2}\right) \\
& \nu=0.3(\cdot) \\
& \mu=0.12(\cdot)
\end{aligned}
$$

